\title{
What role does stereotactic ablative radiotherapy have in advanced castrate-resistant prostate cancer?
}
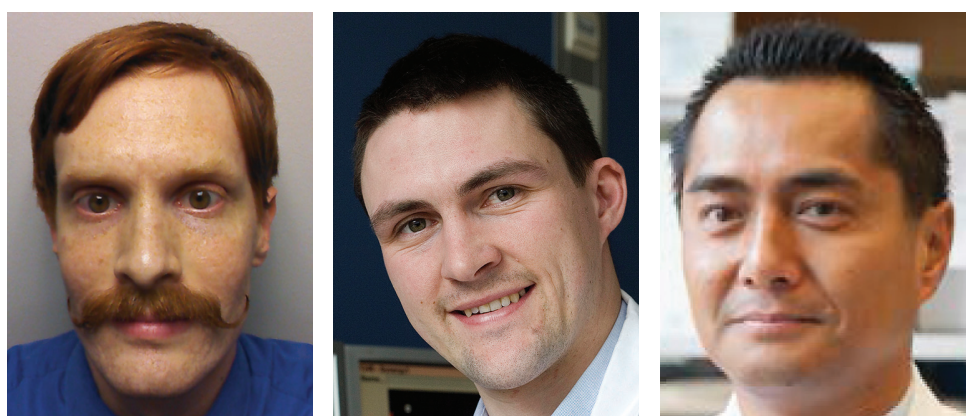

\section{Future ONCOLOGY}

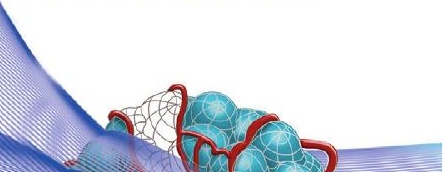

Ryan Phillips', Piet Ost ${ }^{2} \&$ Phuoc T Tran ${ }^{* 1,3}$

First draft submitted: 17 July 2017; Accepted for publication: 20 July 2017;

Published online: 11 October 2017

Prostate cancer is the most commonly diagnosed malignancy in men; the Surveillance, Epidemiology and End Results program estimates over 160,000 estimated new cases and nearly 27,000 deaths from prostate cancer in 2017 [1]. While 10-year prostate cancer specific survival for localized disease is approximately $99 \%$ with either surgical and radiotherapeutic intervention [2], $5 \%$ of men have distant metastatic disease at diagnosis and many more are at risk of developing distant metastases at some point. Historically, metastatic prostate cancer has been considered an incurable disease and the cornerstone of treatment has been androgen deprivation therapy (ADT), a systemic approach that can prolong life substantially, albeit with bothersome side effects such as hot flashes, fatigue, weight gain and muscle wasting [3]. While indefinite ADT will eventually lead to the emergence of castrate-resistant clones that are no longer effectively suppressed by standard first-line therapies, the catalog of effective systemic approaches to forestall progression and prolong life continues to expand [4].

While to date, metastatic prostate cancer remains incurable with a median overall survival of 42 months [5], significant reason for hope remains as the classical management paradigm continues to advance, but also due to mounting evidence that many malignancies including prostate cancer [6] can progress from locally confined to widely metastatic through an intermediate state termed the 'oligometastatic state', proposed by Weichselbaum and Hellman more than 20 years ago [7]. In its original formulation, this state represented a phase of the disease at which some spread beyond the primary had occurred but where local therapy could still be expected to improve disease control. Efforts to define this state have heretofore focused primarily on a clinical definition, analyzing outcomes of patients with a small number of metastases to find a threshold below which superior

\begin{abstract}
"As we continue to advance our ability to detect and characterize metastatic disease, we can make ever more informed treatment decisions..."
\end{abstract}

\section{KEYWORDS}

- castration-resistant prostate cancer - neoplasm metastasis • neoplasm recurrence $\bullet$ oligometastasis $\bullet$ prostate cancer $\bullet$ stereotactic ablative radiotherapy • stereotactic body radiation therapy 


\author{
"Prostate cancer is a \\ relatively late-responding \\ malignancy perhaps better \\ suited to a high \\ dose-per-fraction \\ approach"
}

outcomes were more likely. This numerical cutoff appears to vary based on patient population, available interventions and most importantly underlying disease biology [8].

The paradigm of defining oligometastatic disease based on number of detectable lesions has also been the primary approach in prostate cancer, with thresholds ranging from 3 to 10 lesions [8]. Stereotactic ablative radiotherapy (SABR) has emerged as a promising metastasis-directed therapy (MDT), supported by the radiobiological insight that prostate cancer is a relatively late-responding malignancy perhaps better suited to a high dose-per-fraction approach [9] [Tran PT \& Terezakis SA, Unpublished DATA]. To date, reports of this approach consist primarily of retrospective, single-institution studies. The overwhelming majority of these reports suggest high local control rates with side effects that were mild [10-12]. In addition, in men with hormone-sensitive disease where adjuvant ADT was withheld until evidence of progression, men could be withheld from ADT treatment with a median ADT-free survival of 38 months [13]. Retreatment with SABR was permitted in men who recurred with no more than three new lesions (oligoprogression). Again, no grade 3 or higher toxicities were noted. A subsequent report found that second and third rounds of SABR in men with oligoprogression are similarly safe [13]. The population examined from these retrospective series comprises a highly heterogeneous group and are primarily composed of men with hormone-sensitive disease.

What about metastatic castrate-resistant prostate cancer (mCRPC) that has oligoprogressed on systemic therapy? There are encouraging data for SABR in other aggressive histologies such as nonsmall-cell lung cancer (NSCLC). A randomized, controlled, Phase II study of patients with stage IV NSCLC having $\leq 3$ metastases showed a significant progression free survival (PFS) benefit (HR: 0.35; 90\% CI: 0.18-0.66; log-rank p = 0.0054) in patients receiving consolidative therapy to all lesions as compared with maintenance therapy alone [14]. In addition, Gan et al. observed 6- and 12 -month local control rates of 100 and $86 \%$, respectively, for patients with oligoprogressive $A L K$-rearranged NSCLC, defined as $\leq 4$ metastatic lesions outside the central nervous system, who were receiving crizotinib [15]. Furthermore, these patients remained on crizotinib for nearly three-times as long with suggestion of an associated overall survival benefit. Thus, instead of transitioning to another systemic treatment, SABR may be an option for patients with oligoprogressive disease allowing them to remain on their current systemic therapy. The biological rationale is that the progressing metastases contain resistant clonogens which are a minor fraction of the total disease burden that is otherwise responsive to the current systemic therapy and that ablating these resistant macroscopic metastases can return the patient back to a disease responsive state $[16,17]$. These are just a few representative studies underlining the value of pursuing treatment as early as possible along the metastatic spectrum.

There are promising but very limited data for oligometastatic CRPC (oligometCRPC). First, this population of men with oligometCRPC does exist. Men progressing on ADT or other systemic hormonal therapies often have widespread or polymetastatic disease, however, up to a third of these men have less than three metastases [18]. Triggiani et al. treated 49 lymph node and 21 bone metastases in 41 men with oligometCRPC [19]. After a median follow-up of 24 months, 1 - and 2 -year distant PFS was 43.2 and $21.6 \%$, respectively. The median time to the start of second line systemic treatment was 22 months. Tabata et al. described 35 men, $20 \%$ castrate-resistant, with 6 or fewer osseous metastases identified by bone scan who received SABR [20]. With a median follow-up of 36 months, they observed a 1 -year PFS of $64.8 \%$ and a 3 -year overall survival of $77.2 \%$. All patients in this study received ADT. Univariate analysis suggested a benefit to doses > $40 \mathrm{~Gy}$, however this trend was lost on propensity score analysis adjusting for the heterogeneous population studied. Ahmed et al. reported on SABR in 17 men with $\leq 5$ metastases (71\% had a single lesion and $90 \%$ of lesions were osseous) identified by C-11 choline PET, MRI, or CT [21]. Overall they observed $100 \%$ local control and $71 \%$ biochemical PFS (bPFS) at a median follow-up of 6 months. A total of $65 \%$ of these patients were castrate-resistant and among these, bPFS was $55 \%$ with a median follow-up of 4.8 months. They observed no grade 3 or greater toxicity. In our own recent retrospective analysis of 17 men with oligometCRPC treated with SABR to all macroscopic tumor deposits, median time to next intervention was 45 weeks [22]. Data also suggest that oligometCRPC in the setting of second line hormonal agents such as enzalutamide and abiraterone can also be converted back to a responsive state, which is likely explained by the androgen receptor oncogene-addicted nature 
of prostate cancer [23]. These small reports and other similar studies suggest that MDTs, specifically SABR may be a valuable addition to the armamentarium of advanced prostate cancer management resistant to ADT.

While the data to support SABR for MDT in oligometastatic prostate cancer thus far comprise retrospective reviews, several current prospective trials are underway to better characterize this approach. The Belgian STOMP trial (NCT01558427) has prospectively randomized oligometastatic, hormone-sensitive patients to either MDT (surgery or SABR) or surveillance with a primary end point of ADT-free survival [24]. The Baltimore ORIOLE trial (NCT02680587) is similarly assessing progression in oligometastatic, hormone-sensitive men randomized to SABR or observation [25]. The PCS IX trial seeks to treat oligometastatic, castrate-resistant patients with standard ADT and enzalutamide, plus randomization to SABR or no MDT (NCT02685397).

To date, efforts to advance SABR as MDT in oligometastatic prostate cancer have shown excellent local control rates and promising PFS, but have not yet translated to significant cure rates. One compelling explanation is that the attempts to define the oligometastatic state by counting lesions will always be limited by the sensitivity and specificity of the detection method and likely by patient characteristics that are not well understood. As newer molecular imaging modalities such as prostate specific membrane antigentargeted imaging mature, the value of MDT in the oligometastatic state may prove to be a moving target as patients previously defined as oligometastatic based on conventional imaging may have additional detectable tumor deposits and require reclassification as polymetastatic [26].

Ultimately, we believe an approach to define the oligometastatic state by examining disease biology will be the most accurate. For prostate cancer this means answering such critical questions as: 'What restricts prostate cancer in some men to a few sites, while allowing widespread dissemination in others'?; 'Among men with oligometastatic prostate cancer, is there a threshold of systemic disease burden below which the disease can no longer sustain itself and might be curable'?; and, 'Do germline and somatic mutations in DNA repair genes modify the metastatic state and response to MDTs such as SABR'?. While these questions remain unanswered to date, a fundamental understanding of this concept may allow stratification of men into those for whom local consolidation of all macroscopic tumor deposits would reasonably be expected to provide hope for a cure, and those for whom early systemic therapy is necessary to forestall disease progression. The Movember Foundation is set to launch their Global Action Plan 6 initiative on oligometastatic prostate cancer with one major goal set to tackle understanding the biological differences between hormone-sensitive oligometastatic and polymetastatic prostate cancer at the genetic and transcriptomic level [27].

Promising attempts to elucidate the biological underpinnings of metastasis, and by extension the oligometastatic state, include but are not limited to studies of circulating tumor cells, circulating tumor DNA, investigation of mutations predisposing to metastasis and histologic subtypes of prostate cancer that may behave differently with respect to systemic dissemination and response to therapies. As we continue to advance our ability to detect and characterize metastatic disease, we can make ever more informed treatment decisions and maximally leverage all available treatment modalities in this highly prevalent and potentially life-threatening disease. In closing, there are mounting positive retrospective data for hormone sensitive oligometastatic prostate cancer and encouraging but still preliminary data with oligometCRPC that SABR can have a role in advanced disease. We encourage basic and clinical efforts to further validate and refine that role.

Financial \& competing interests disclosure

$P$ Ost is a senior clinical investigator of the Research Foundation - Flanders (FWO) (Belgium). PT Tran is funded by the Nesbitt-McMaster Foundation, American Lung Association (LCD-339465), Movember-PCF and the NIH (R01CA166348 \& U01CA183031); PT Tran owns the patent "Compounds and Methods of Use in Ablative Radiotherapy. Patent filed 3/9/2012. PCT/US2012/028475. PCT/WO/2012/122471. Patent awarded 5/25/2015. Patient\#: 9114158 ." The authors have no other relevant affiliations or financial involvement with any organization or entity with a financial interest in or financial conflict with the subject matter or materials discussed in the manuscript apart from those disclosed.

No writing assistance was utilized in the production of this manuscript.

\section{Open access}

This work is licensed under the AttributionNonCommercial-NoDerivatives 4.0 Unported License. To view a copy of this license, visit http://creativecommons.org/ licenses/by-nc-nd/4.0/
“. . attempts to define the oligometastatic state by counting lesions will always be limited by the sensitivity and specificity of the detection method..." 


\section{References}

1 Cancer stat facts: prostate cancer. Surveillance, Epidemiology and End Results (SEER) (2017). https://seer.cancer.gov/statfacts/html/

2 Hamdy FC, Donovan JL, Lane JA et al. 10 -year outcomes after monitoring, surgery or radiotherapy for localized prostate cancer. N. Engl. J. Med. 375(15), 1415-1424 (2016).

3 Litwin MS, Tan HJ. The diagnosis and treatment of prostate cancer: a review. JAMA 317(24), 2532-2542 (2017).

4 Handy CE, Antonarakis ES. Sequencing treatment for castration-resistant prostate cancer. Curr. Treat. Options Oncol. 17(12), 64 (2016).

5 James ND, Spears MR, Clarke NW et al. Survival with newly diagnosed metastatic prostate cancer in the "Docetaxel Era": data from 917 patients in the control arm of the STAMPEDE trial (MRC PR08, CRUK/06/019). Eur. Urol. 67(6), 1028-1038 (2015).

6 Tran PT, Antonarakis ES. Altering the natural history of oligometastatic prostate cancer with local therapies: reality versus illusion. J. Oncol. Pract. 13(1), 21-24 (2017).

7 Hellman S, Weichselbaum RR. Oligometastases. J. Clin. Oncol. 13(1), 8-10 (1995).

8 Tosoian JJ, Gorin MA, Ross AE, Pienta KJ, Tran PT, Schaeffer EM. Oligometastatic prostate cancer: definitions, clinical outcomes and treatment considerations. Nat. Rev. Urol. 14(1), 15-25 (2017).

9 Nahum AE. The radiobiology of hypofractionation. Clin. Oncol. 27(5), 260-269 (2015).

10 Muacevic A, Kufeld M, Rist C, Wowra B, Stief C, Staehler M. Safety and feasibility of image-guided robotic radiosurgery for patients with limited bone metastases of prostate cancer. Urol. Oncol. 31(4), 455-460 (2013).
11 Schick U, Jorcano S, Nouet P et al. Androgen deprivation and high-dose radiotherapy for oligometastatic prostate cancer patients with less than five regional and/or distant metastases. Acta. Oncol. 52(8), 1622-1628 (2013).

12 Berkovic P, De Meerleer G, Delrue L et al. Salvage stereotactic body radiotherapy for patients with limited prostate cancer metastases: deferring androgen deprivation therapy. Clin. Genitourin. Cancer 11(1), 27-32 (2013).

13 Decaestecker K, De Meerleer G, Lambert B et al. Repeated stereotactic body radiotherapy for oligometastatic prostate cancer recurrence. Radiat. Oncol. 9, 135 (2014).

14 Gomez DR, Blumenschein GR Jr, Lee JJ et al. Local consolidative therapy versus maintenance therapy or observation for patients with oligometastatic non-small-cell lung cancer without progression after first-line systemic therapy: a multicentre, randomized, controlled, Phase II study. Lancet Oncol. 17(12), 1672-1682 (2016).

15 Gan GN, Weickhardt AJ, Scheier B et al. Stereotactic radiation therapy can safely and durably control sites of extra-central nervous system oligoprogressive disease in anaplastic lymphoma kinase-positive lung cancer patients receiving crizotinib. Int. J. Radiat. Oncol. Biol. Phys. 88(4), 892-898 (2014).

16 Gundem G, Van Loo P, Kremeyer B et al. The evolutionary history of lethal metastatic prostate cancer. Nature 520(7547), 353-357 (2015).

17 Hong MK, Macintyre G, Wedge DC et al. Tracking the origins and drivers of subclonal metastatic expansion in prostate cancer. Nat Commun. 6, 6605 (2015).

18 Mcdonald E, Cheng S, Arciero V et al. Prevalence of oligoprogressive, metastatic castration-resistant prostate cancer (mCRPC) amenable to stereotactic ablative radiotherapy $(\mathrm{SABR})$ in men undergoing abiraterone acetate (AA) therapy. Presented at: ASCO $G U$. Orlando, FL, USA, 16 February 2017.
19 Triggiani L, Alongi F, Buglione M et al. Efficacy of stereotactic body radiotherapy in oligorecurrent and in oligoprogressive prostate cancer: new evidence from a multicentric study. Br. J. Cancer 116(12), 1520-1525 (2017).

20 Tabata K, Niibe Y, Satoh T et al. Radiotherapy for oligometastases and oligorecurrence of bone in prostate cancer. Pulm. Med. 2012, 541656 (2012).

21 Ahmed KA, Barney BM, Davis BJ, Park SS, Kwon ED, Olivier KR. Stereotactic body radiation therapy in the treatment of oligometastatic prostate cancer. Front. Oncol. 2, 215 (2012).

22 Tran PT, Moyer CL, Phillips R et al. Stereotactic ablative radiation therapy for the treatment of oligometastatic prostate cancer. J. Clin. Oncol. 35(Suppl. 15), Abstract 5020 (2017).

23 Coutinho I, Day TK, Tilley WD, Selth LA. Androgen receptor signaling in castration-resistant prostate cancer: a lesson in persistence. Endocr. Relat. Cancer 23(12), T179-T197 (2016).

24 Decaestecker K, De Meerleer G, Ameye F et al. Surveillance or metastasis-directed therapy for oligometastatic prostate cancer recurrence (STOMP): study protocol for a randomized Phase II trial. BMC Cancer 14, 671 (2014).

25 Radwan N, Phillips R, Ross A et al. A Phase II randomized trial of Observation versus stereotactic ablative RadiatIon for OLigometastatic prostate CancEr (ORIOLE). BMC Cancer 17(1), 453 (2017).

26 Rowe SP, Gorin MA, Allaf ME et al. PET imaging of prostate-specific membrane antigen in prostate cancer: current state of the art and future challenges. Prostate Cancer Prostatic Dis. 19(3), 223-230 (2016).

27 Movember Gobal Action Plan 6. The Movember Foundation (2017). https://gap6.fluidreview.com/ 